Bull. Korean Math. Soc. 50 (2013), No. 6, pp. 1817-1826

http://dx.doi.org/10.4134/BKMS.2013.50.6.1817

\title{
EXISTENCE OF THREE SOLUTIONS FOR A NAVIER BOUNDARY VALUE PROBLEM INVOLVING THE $p(x)$-BIHARMONIC
}

\author{
Honghui Yin AND Ying LiU
}

\begin{abstract}
The existence of at least three weak solutions is established for a class of quasilinear elliptic equations involving the $p(x)$-biharmonic operators with Navier boundary value conditions. The technical approach is mainly based on a three critical points theorem due to Ricceri [11].
\end{abstract}

\section{Introduction}

In this paper, we consider the problem of the type

$$
\left\{\begin{array}{l}
\triangle_{p(x)}^{2} u=\lambda a(x) f(x, u)+\mu g(x, u), \quad x \in \Omega \\
u=\triangle u=0, \quad x \in \partial \Omega
\end{array}\right.
$$

where $\Omega \subset \mathbb{R}^{N}(N \geq 2)$ is a bounded domain with boundary of class $C^{1}, \lambda, \mu \geq$ 0 are real numbers, $p(x) \in C^{0}(\bar{\Omega})$ with $\max \left\{2, \frac{N}{2}\right\}<p^{-}:=\inf _{x \in \bar{\Omega}} p(x) \leq$ $p^{+}:=\sup _{x \in \bar{\Omega}} p(x), \triangle_{p(x)}^{2}:=\triangle\left(|\triangle u|^{p(x)-2} \triangle u\right)$ is the operator of fourth order called the $p(x)$-biharmonic operator, which is a natural generalization of the $p$-biharmonic operator (where $p>1$ is a constant).

In [10], the authors studied the following super-linear $p$-biharmonic elliptic problem with Navier boundary conditions:

$$
\left\{\begin{array}{l}
\triangle_{p}^{2} u=g(x, u), \quad x \in \Omega \\
u=\triangle u=0, \quad x \in \partial \Omega
\end{array}\right.
$$

By means of Morse theory, the authors proved the existence of a nontrivial solution to (2) having a linking structure around the origin under the conditions: $\Omega \subseteq \mathbb{R}^{N}$ is bounded with smooth boundary, $N \geq 2 p+1, g: \Omega \times \mathbb{R} \rightarrow \mathbb{R}$ is a Carathéodory function such that for some $C>0,|g(x, t)| \leq C\left(1+|t|^{q-1}\right)$ for a.e. $x \in \Omega$ and all $t \in \mathbb{R}, 1 \leq q \leq p^{*}=\frac{N p}{N-2 p}$. Moreover, in case of both

Received October 9, 2011; Revised November 13, 2012.

2010 Mathematics Subject Classification. 35D05, 35J60.

Key words and phrases. $p(x)$-biharmonic, three solutions, existence.

Project Supported by the Natural Science Foundation of Jiangsu Education Office (No.12KJB110002). 
resonance near zero and non-resonance at $\infty$, the existence of two nontrivial solutions was obtained.

In [9], the authors considered the following problem:

$$
\left\{\begin{array}{l}
\triangle_{p}^{2} u=\lambda f(x, u)+\mu g(x, u), \quad x \in \Omega \\
u=\triangle u=0, \quad x \in \partial \Omega
\end{array}\right.
$$

By the three critical points theorem obtained by Ricceri [11], they established the existence of three weak solutions to problem (3).

For more results for fourth-order elliptic equations with variable exponent, see $[1,2]$ and the reference therein.

To obtain the existence of at least three solutions of problem (1), the technical approach is mainly based on a three critical points theorem by B. Ricceri [11].

Theorem A. Let $X$ be a reflexive real Banach space; $I \subseteq \mathbb{R}$ an interval; $\Phi: X \rightarrow \mathbb{R}$ a continuously Gâteaux differentiable and sequentially weakly lower semicontinuous $C^{1}$ functional, bounded on each bounded subset of $X$, whose Gâteaux derivative admits a continuous inverse on $X^{*} ; \Psi: X \rightarrow \mathbb{R}$ a $C^{1}$ functional with compact Gâteaux derivative. Assume that

(i) $\lim _{\|u\| \rightarrow \infty}(\Phi(u)+\lambda \Psi(u))=\infty$ for all $\lambda \in I$;

(ii) There exists $\rho \in \mathbb{R}$ such that:

$$
\sup _{\lambda \in I} \inf _{t \in X}(\Phi(t)+\lambda(\Psi(t)+\rho))<\inf _{t \in X} \sup _{\lambda \in I}(\Phi(t)+\lambda(\Psi(t)+\rho)) .
$$

Then there exists a non-empty open set $\Lambda \subseteq I$ and a positive real number $\sigma$ with the following property: for each $\lambda \in \Lambda$ and every $C^{1}$ functional $J: X \rightarrow \mathbb{R}$ with compact Gâteaux derivative, there exists $\delta>0$ such that for each $\mu \in[0, \delta]$, the equation

$$
\Phi^{\prime}(u)+\lambda \Psi^{\prime}(u)+\mu J^{\prime}(u)=0
$$

has at least three solutions in $X$ whose norms are less than $\sigma$.

To obtain the existence of at least three solutions of (1), we assume the following conditions:

(A) $g: \Omega \times \mathbb{R} \rightarrow \mathbb{R}$ is a Carathéodory function, $\sup _{|\zeta| \leq s}|g(\cdot, \zeta)| \in L^{1}(\Omega)$ for all $s>0$;

(B) $a(x) \in L^{r(x)}(\Omega), f: \Omega \times \mathbb{R} \rightarrow \mathbb{R}$ is a Carathéodory function, $|f(x, t)| \leq$ $b(x)+\alpha|t|^{q(x)-1}$ for $x \in \Omega$ and $t \in \mathbb{R}$, where $\alpha \geq 0$ is a constant, $b(x) \in$ $L^{q^{0}(x) r^{0}(x)}(\Omega), r(x), q(x) \in C(\Omega), r^{-}>1, p^{-}>q^{+} \geq q^{-} \geq 1$, and

$$
q(x)<\frac{r(x)-1}{r(x)} p^{*}(x), \quad \forall x \in \Omega,
$$

here

$$
p^{*}(x)=\left\{\begin{array}{l}
\frac{N p(x)}{N-p(x)}, \quad p(x)<N \\
\infty, \quad p(x) \geq N
\end{array}\right.
$$

and $r^{0}(x)$ is the conjugate function of $r(x)$, i.e., $\frac{1}{r(x)}+\frac{1}{r^{0}(x)}=1$. 
The paper is organized as follows. In Section 2, we recall some facts that will be needed in the paper. In Section 3, we establish our main results.

\section{Notations and preliminaries}

For the reader's convenience, we remind some background facts concerning the Lebesgue-Sobolev spaces with variable exponent and introduce some notations used below. For more details, we refer the reader to $[5,7,12,13]$.

Set

$$
C_{+}(\Omega)=\{h: h \in C(\bar{\Omega}) \text { and } h(x)>1 \text { for all } x \in \bar{\Omega}\} .
$$

For $p(x) \in C_{+}(\Omega)$, define the space

$L^{p(x)}(\Omega)=\left\{u \mid u\right.$ is a measurable real-valued funcion, $\left.\int_{\Omega}|u(x)|^{p(x)} d x<\infty\right\}$.

We can introduce a norm on $L^{p(x)}(\Omega)$ by

$$
|u|_{p(x)}=\inf \left\{\lambda>\left.0\left|\int_{\Omega}\right| \frac{u(x)}{\lambda}\right|^{p(x)} d x \leq 1\right\} .
$$

and $\left(L^{p(x)}(\Omega),|\cdot|_{p(x)}\right)$ becomes a Banach space, and we call it variable exponent Lebesgue space.

The space $W^{m, p(x)}(\Omega)$ is defined by

$$
W^{m, p(x)}(\Omega)=\left\{u \in L^{p(x)}(\Omega)\left|D^{\beta} u \in L^{p(x)}(\Omega),\right| \beta \mid \leq m\right\},
$$

where $\beta$ is the multi-index and $|\beta|$ is the order, $m$ is a positive integer.

$$
W^{m, p(x)}(\Omega)
$$

is a special class of so-called generalized Orlicz-Sobolev spaces. From [6], we know that $W^{m, p(x)}(\Omega)$ can be equipped with the norm $\|u\|_{W^{m, p(x)}(\Omega)}$ as Banach spaces, where

$$
\|u\|_{W^{m, p(x)}(\Omega)}=\sum_{|\beta| \leq m}\left|D^{\alpha} u\right|_{p(x)} .
$$

From [5], we know that spaces $L^{p(x)}(\Omega)$ and $W^{m, p(x)}(\Omega)$ are separable, reflexive and uniform convex Banach spaces.

Now we denote $X=W^{2, p(x)}(\Omega) \cap W_{0}^{1, p(x)}(\Omega)$, where $W_{0}^{1, p(x)}(\Omega)$ denote the closure of $C_{0}^{\infty}(\Omega)$ in $W^{1, p(x)}(\Omega)$. For any $u \in X$, define

$$
\|u\|=\inf \left\{\lambda>\left.0\left|\int_{\Omega}\right| \frac{\triangle u(x)}{\lambda}\right|^{p(x)} d x \leq 1\right\} .
$$

Then it is easy to see that $X$ endowed with the above norm is also a separable, reflexive Banach space. We denote by $X^{*}$ the dual space to $X$.

Remark 2.1. According to [14], $\|u\|_{W^{2, p(x)}(\Omega)}$ is equivalent to $|\Delta u|_{p(x)}$ in $X$. Consequently, the norms $\|u\|_{W^{2, p(x)}(\Omega)}$ and $\|u\|$ are equivalent.

From now on, we will use $\|\cdot\|$ instead of $\|\cdot\|_{W^{2, p(x)}(\Omega)}$ on $X$. 
Proposition 2.1 (see $[5,12]$ ). The conjugate space of $L^{p(x)}(\Omega)$ is $L^{p^{0}(x)}(\Omega)$. For any $u \in L^{p(x)}(\Omega)$ and $v \in L^{p^{0}(x)}(\Omega)$, we have

$$
\int_{\Omega}|u v| d x \leq\left(\frac{1}{p^{-}}+\frac{1}{\left(p^{0}\right)^{-}}\right)|u|_{p(x)}|v|_{p^{0}(x)} \leq 2|u|_{p(x)}|v|_{p^{0}(x)} .
$$

Proposition 2.2 (see $[5,12])$. If we denote $\rho(u)=\int_{\Omega}|u|^{p(x)} d x, \forall u \in L^{p(x)}(\Omega)$, then

(i) $|u|_{p(x)}<1(=1 ;>1) \Leftrightarrow \rho(u)<1(=1 ;>1)$;

(ii) $|u|_{p(x)}>1 \Rightarrow|u|_{p(x)}^{p^{-}} \leq \rho(u) \leq|u|_{p(x)}^{p^{+}} ;|u|_{p(x)}<1 \Rightarrow|u|_{p(x)}^{p^{+}} \leq \rho(u) \leq$ $|u|_{p(x)}^{p^{-}}$

(iii) $|u|_{p(x)} \rightarrow 0(\infty) \Leftrightarrow \rho(u) \rightarrow 0(\infty)$.

From Proposition 2.2, the following inequalities hold:

$$
\begin{gathered}
\|u\|^{p^{-}} \leq \int_{\Omega}|\triangle u(x)|^{p(x)} d x \leq\|u\|^{p^{+}}, \text {if }\|u\| \geq 1 ; \\
\|u\|^{p^{+}} \leq \int_{\Omega}|\triangle u(x)|^{p(x)} d x \leq\|u\|^{p^{-}}, \text {if }\|u\| \leq 1 .
\end{gathered}
$$

Proposition 2.3 (see [4]). Suppose that the boundary of $\Omega$ possesses the cone property and $a(x) \in L^{r(x)}(\Omega), a(x)>0$ for a.e. $x \in \Omega, r(x) \in C(\bar{\Omega})$ and $r^{-}>1$. If $p(x), q(x) \in C(\bar{\Omega})$ and

$$
1 \leq q(x)<\frac{r(x)-1}{r(x)} p^{*}(x), \quad \forall x \in \bar{\Omega},
$$

then there is a compact embedding $X \hookrightarrow L_{a(x)}^{q(x)}(\Omega)$.

Proposition 2.4. If $\Omega \subset R^{N}$ is a bounded domain, then the imbedding $X \hookrightarrow$ $C^{0}(\bar{\Omega})$ is compact whenever $\frac{N}{2}<p^{-}$.

Proof. It is well known that $X \hookrightarrow W^{2, p^{-}}(\Omega) \cap W_{0}^{1, p^{-}}(\Omega)$ is a continuous embedding, and the embedding $W^{2, p^{-}}(\Omega) \cap W_{0}^{1, p^{-}}(\Omega) \hookrightarrow C^{0}(\bar{\Omega})$ is compact when $\frac{N}{2}<p^{-}$and $\Omega$ is bounded. So we obtain the embedding $X \hookrightarrow C^{0}(\bar{\Omega})$ which is compact whenever $\frac{N}{2}<p^{-}$.

From Proposition 2.4, there exists a positive constant $k$ depending on $p(x)$, $N$ and $\Omega$, such that

$$
\|u\|_{\infty}=\sup _{x \in \bar{\Omega}}|u(x)| \leq k\|u\|, \forall u \in X .
$$

\section{Existence of three solutions}

Fix $x^{0} \in \Omega$ and choose $r_{1}, r_{2}$ with $0<r_{1}<r_{2}$, such that $B\left(x^{0}, r_{2}\right) \subseteq \Omega$, where $B(x, r)$ stands for the open ball in $\mathbb{R}^{N}$ of radius $r$ centered at $x$. Let

$$
\sigma=\max \left\{\left[\frac{12(N+2)^{2}\left(r_{1}+r_{2}\right)}{\left(r_{2}-r_{1}\right)^{3}}\right]^{p^{-}} \frac{2 \pi^{\frac{N}{2}}\left(r_{2}^{N}-r_{1}^{N}\right)}{N \Gamma\left(\frac{N}{2}\right)},\left[\frac{12(N+2)^{2}\left(r_{1}+r_{2}\right)}{\left(r_{2}-r_{1}\right)^{3}}\right]^{p^{+}} \frac{2 \pi^{\frac{N}{2}}\left(r_{2}^{N}-r_{1}^{N}\right)}{N \Gamma\left(\frac{N}{2}\right)}\right\},
$$


$\theta=\left\{\begin{array}{l}\min \left\{\left[\frac{3 N}{\left(r_{2}-r_{1}\right)\left(r_{2}+r_{1}\right)}\right]^{p^{-}} \frac{2 \pi^{\frac{N}{2}}\left[\left(r_{2}+r_{1}\right)^{N}-\left(2 r_{1}\right)^{N}\right]}{2^{N} N \Gamma\left(\frac{N}{2}\right)},\left[\frac{3 N}{\left(r_{2}-r_{1}\right)\left(r_{2}+r_{1}\right)}\right]^{p^{+}} \frac{2 \pi^{\frac{N}{2}}\left[\left(r_{2}+r_{1}\right)^{N}-\left(2 r_{1}\right)^{N}\right]}{2^{N} N \Gamma\left(\frac{N}{2}\right)}\right\}, \\ \text { if } N<\frac{4 r_{1}}{r_{2}-r_{1}}, \\ \min \left\{\left[\frac{12 r_{1}}{\left(r_{2}-r_{1}\right)^{2}\left(r_{2}+r_{1}\right)}\right]^{p^{-}} \frac{2 \pi^{\frac{N}{2}}\left[\left(r_{2}+r_{1}\right)^{N}-\left(2 r_{1}\right)^{N}\right]}{2^{N} N \Gamma\left(\frac{N}{2}\right)},\left[\frac{12 r_{1}}{\left(r_{2}-r_{1}\right)^{2}\left(r_{2}+r_{1}\right)}\right]^{p^{+}} \frac{2 \pi^{\frac{N}{2}}\left[\left(r_{2}+r_{1}\right)^{N}-\left(2 r_{1}\right)^{N}\right]}{2^{N} N \Gamma\left(\frac{N}{2}\right)}\right\}, \\ \text { if } N \geq \frac{4 r_{1}}{r_{2}-r_{1}} .\end{array}\right.$

We define $\Phi: X \rightarrow \mathbb{R}$ as

$$
\Phi(u)=\int_{\Omega} \frac{1}{p(x)}|\triangle u(x)|^{p(x)} d x .
$$

Then

Denote

$$
\left(\Phi^{\prime}(u), v\right)=\int_{\Omega}|\triangle u|^{p(x)-2} \triangle u \triangle v d x, \quad \forall u, v \in X .
$$

$$
\begin{aligned}
F(x, u) & =\int_{0}^{u} a(x) f(x, t) d t, G(x, u)=\int_{0}^{u} g(x, t) d t \\
\Psi(u) & =-\int_{\Omega} F(x, u) d x, J(u)=-\int_{\Omega} G(x, u) d x .
\end{aligned}
$$

Then for $\forall u, v \in X$,

$$
\begin{aligned}
& \left(\Psi^{\prime}(u), v\right)=-\int_{\Omega} a(x) f(x, u) v d x \\
& \left(J^{\prime}(u), v\right)=-\int_{\Omega} g(x, u) v d x .
\end{aligned}
$$

We say that $u \in X$ is a weak solution of problem (1) if

$$
\int_{\Omega}|\triangle u|^{p(x)-2} \triangle u \triangle v d x=\lambda \int_{\Omega} a(x) f(x, u) v d x+\mu \int_{\Omega} g(x, u) v d x, \forall v \in X,
$$

i.e.,

$$
\left(\Psi^{\prime}(u), v\right)+\lambda\left(\Psi^{\prime}(u), v\right)+\mu\left(J^{\prime}(u), v\right)=0 .
$$

It follows that we can find the weak solutions of (1) applying Theorem A.

We first obtain the following results.

Lemma 3.1. If $\Phi$ is defined in (8), then $\left(\Phi^{\prime}\right)^{-1}: X^{*} \rightarrow X$ exists and it is continuous.

Proof. First, we show that $\Phi^{\prime}$ is uniformly monotone. In fact, for any $\zeta, \eta \in R^{N}$, we have the following inequality (see [8]):

$$
\left(|\zeta|^{p-2} \zeta-|\eta|^{p-2} \eta\right)(\zeta-\eta) \geq \frac{1}{2^{p}}|\zeta-\eta|^{p}, p \geq 2
$$

Thus, we deduce that

$$
\left(\Phi^{\prime}(u)-\Phi^{\prime}(v), u-v\right) \geq \frac{1}{2^{p^{+}}} \int_{\Omega}|\triangle u-\triangle v|^{p(x)} d x, \forall u, v \in X,
$$

i.e., $\Phi^{\prime}$ is uniformly monotone. 
From (5), we can see that for any $u \in X$ with $\|u\| \geq 1$, and hence we have that

$$
\frac{\left(\Phi^{\prime}(u), u\right)}{\|u\|} \geq\|u\|^{p^{-}-1}
$$

This means that $\Phi^{\prime}$ is coercive on $X$.

By a standard argument, we know that $\Phi^{\prime}$ is hemicontinuous. Therefore, the conclusion follows immediately by applying Theorem 26.A [15].

Lemma 3.2. If condition (B) holds, then for any $\lambda \in R, \Phi(u)+\lambda \Psi(u)$ is coercive on $X$.

Proof. For $|f(x, t)| \leq b(x)+\alpha|t|^{q(x)-1}$ and the Young's inequality, we have that

$$
|F(x, t)| \leq|a(x)|\left(b(x)|t|+\frac{\alpha}{q(x)}|t|^{q(x)}\right) \leq|a(x)|\left((b(x))^{q^{0}(x)}+(1+\alpha)|t|^{q(x)}\right) .
$$

Then from condition (B) and Proposition 2.3 we know that $F(x, u)$ is integrable on $\Omega$ for any $u \in X, \Psi(u)$ is well defined.

Combining it with Proposition 2.3, we have

$$
\begin{aligned}
& \Phi(u)+\lambda \Psi(u) \\
= & \int_{\Omega} \frac{1}{p(x)}|\nabla u(x)|^{p(x)} d x-\lambda \int_{\Omega} F(x, u) d x \\
\geq & \frac{\|u\|^{p^{-}}}{p^{+}}-|\lambda| \int_{\Omega}|a(x)|\left[|b(x)|^{q^{0}(x)}+(1+\alpha)|u|^{q(x)}\right] d x \\
\geq & \frac{\|u\|^{p^{-}}}{p^{+}}-|\lambda| C_{1}-|\lambda|(1+\alpha)|u|_{(q(x),|a(x)|)}^{q^{\dagger}} \\
\geq & \frac{\|u\|^{p^{-}}}{p^{+}}-|\lambda| C_{1}-|\lambda| C_{2}\|u\|^{q^{\dagger}}
\end{aligned}
$$

where

$$
\dagger=\left\{\begin{array}{l}
+, \text { if }|u|_{q(x),|a(x)|} \leq 1 \\
-, \text { if }|u|_{q(x),|a(x)|} \geq 1
\end{array}\right.
$$

and $C_{1}, C_{2}$ are positive constants. Since $q^{\dagger}<p^{-}$, we can see that $\Phi(u)+\lambda \Psi(u)$ is coercive.

Furthermore, we suppose

(C) There exist two positive constants $c, d$ with $k>c$ and $k \theta \min \left\{d^{p^{+}}, d^{p^{-}}\right\}$ $>c$ such that $F(x, t) \geq 0$ for each $(x, t) \in\left\{\Omega \backslash B\left(x^{0}, r_{1}\right)\right\} \times[0, d]$, and

$$
m(\Omega) \max _{(x, t) \in \bar{\Omega} \times[-c, c]} F(x, t) \leq \frac{p^{-}}{p^{+} \sigma \max \left\{d^{p^{+}}, d^{p^{-}}\right\}}\left(\frac{c}{k}\right)^{p^{+}} \int_{B\left(x^{0}, r_{1}\right)} F(x, d) d x,
$$

where $m(\Omega)$ is the Lebesgue measure of $\Omega$.

Then we have the following result. 
Lemma 3.3. If condition (C) holds, then there exist $r>0$ and $u^{*} \in X$ such that

$$
\Phi\left(u^{*}\right)>r
$$

and

$$
m(\Omega) \max _{(x, t) \in \bar{\Omega} \times[-c, c]} F(x, t) \leq r p^{-} \frac{\int_{\Omega} F\left(x, u^{*}\right) d x}{\int_{\Omega}|\triangle u(x)|^{p(x)} d x} .
$$

Proof. Let us define

$$
u^{*}(x)=\left\{\begin{array}{l}
0, \quad x \in \bar{\Omega} \backslash B\left(x^{0}, r_{2}\right), \\
\frac{d\left[3\left(l^{4}-r_{2}^{4}\right)-4\left(r_{1}+r_{2}\right)\left(l^{3}-r_{2}^{3}\right)+6 r_{1} r_{2}\left(l^{2}-r_{2}^{2}\right)\right]}{\left(r_{2}-r_{1}\right)^{3}\left(r_{1}+r_{2}\right)}, x \in B\left(x^{0}, r_{2}\right) \backslash B\left(x^{0}, r_{1}\right), \\
d, \quad x \in B\left(x^{0}, r_{1}\right),
\end{array}\right.
$$

where $l=\operatorname{dist}\left(x, x^{0}\right)=\sqrt{\sum_{i=1}^{N}\left(x_{i}-x_{i}^{0}\right)^{2}}$. Then, we have

$$
\begin{aligned}
& \frac{\partial u^{*}(x)}{\partial x_{i}}=\left\{\begin{array}{l}
0, x \in \bar{\Omega} \backslash B\left(x^{0}, r_{2}\right) \cup B\left(x^{0}, r_{1}\right), \\
\frac{12 d\left[l^{2}\left(x_{i}-x_{i}^{0}\right)-\left(r_{1}+r_{2}\right) l\left(x_{i}-x_{i}^{0}\right)+r_{1} r_{2}\left(x_{i}-x_{i}^{0}\right)\right]}{\left(r_{2}-r_{1}\right)^{3}\left(r_{1}+r_{2}\right)}, x \in B\left(x^{0}, r_{2}\right) \backslash B\left(x^{0}, r_{1}\right),
\end{array}\right. \\
& \frac{\partial^{2} u^{*}(x)}{\partial x_{i}^{2}}=\left\{\begin{array}{l}
0, x \in \bar{\Omega} \backslash B\left(x^{0}, r_{2}\right) \cup B\left(x^{0}, r_{1}\right), \\
\frac{12 d\left[r_{1} r_{2}+\left(2 l-r_{1}-r_{2}\right)\left(x_{i}-x_{i}^{0}\right)^{2} / l-\left(r_{1}+r_{2}-l\right) l\right]}{\left(r_{2}-r_{1}\right)^{3}\left(r_{1}+r_{2}\right)}, x \in B\left(x^{0}, r_{2}\right) \backslash B\left(x^{0}, r_{1}\right),
\end{array}\right.
\end{aligned}
$$

and

$$
\sum_{i=1}^{N} \frac{\partial^{2} u^{*}(x)}{\partial x_{i}^{2}}=\left\{\begin{array}{l}
0, x \in \bar{\Omega} \backslash B\left(x^{0}, r_{2}\right) \cup B\left(x^{0}, r_{1}\right), \\
\frac{12 d\left[(N+2) l^{2}-(N+1)\left(r_{1}+r_{2}\right) l+N r_{1} r_{2}\right]}{\left(r_{2}-r_{1}\right)^{3}\left(r_{1}+r_{2}\right)}, x \in B\left(x^{0}, r_{2}\right) \backslash B\left(x^{0}, r_{1}\right) .
\end{array}\right.
$$

It is easy to verify that $u^{*} \in X$ and, in particular, we have

$$
\theta \min \left\{d^{p^{+}}, d^{p^{-}}\right\} \leq \int_{\Omega}\left|\triangle u^{*}\right|^{p(x)} d x \leq \sigma \max \left\{d^{p^{+}}, d^{p^{-}}\right\}
$$

If we let

$$
r=\frac{1}{p^{+}}\left(\frac{c}{k}\right)^{p^{+}}
$$

from (11) and the assumption that $k \theta \min \left\{d^{p^{+}}, d^{p^{-}}\right\}>c$, we have

$$
\begin{aligned}
\Phi\left(u^{*}\right) & =\int_{\Omega} \frac{1}{p(x)}\left|\triangle u^{*}(x)\right|^{p(x)} d x \\
& \geq \frac{1}{p^{+}} \int_{\Omega}\left|\triangle u^{*}(x)\right|^{p(x)} d x \\
& \geq \frac{1}{p^{+}} \theta \min \left\{d^{p^{+}}, d^{p^{-}}\right\} \\
& >\frac{1}{p^{+}}\left(\frac{c}{k}\right)^{p^{+}}=r .
\end{aligned}
$$

Therefore, (9) follows. 
Since $0 \leq u^{*} \leq d$ for any $x \in \Omega$, the condition (C) ensures that

$$
\int_{\Omega \backslash B\left(x^{0}, r_{2}\right)} F\left(x, u^{*}\right) d x+\int_{B\left(x^{0}, r_{2}\right) \backslash B\left(x^{0}, r_{1}\right)} F\left(x, u^{*}\right) d x \geq 0 .
$$

Therefore, we have

$$
\begin{aligned}
m(\Omega) \max _{(x, t) \in \bar{\Omega} \times[-c, c]} F(x, t) & \leq \frac{p^{-}}{p^{+} \sigma \max \left\{d^{p^{+}}, d^{p^{-}}\right\}}\left(\frac{c}{k}\right)^{p^{+}} \int_{B\left(x^{0}, r_{1}\right)} F(x, d) d x \\
& \leq r p^{-} \frac{\int_{B\left(x^{0}, r_{1}\right)} F(x, d) d x}{\int_{\Omega}|\triangle u(x)|^{p(x)} d x} \\
& \leq r p^{-} \frac{\int_{\Omega} F\left(x, u^{*}\right) d x}{\int_{\Omega}|\triangle u(x)|^{p(x)} d x} .
\end{aligned}
$$

This implies (10).

Finally, we have the following main theorem.

Theorem 3.1. Assume conditions (A), (B) and (C) hold. Then there exist a non-empty open set $\Lambda \subseteq R$ and a positive real number $\sigma$ with the following property: for each $\lambda \in \Lambda$, there exists $\delta>0$ such that for each $\mu \in[0, \delta]$, problem (1) has at least three weak solutions whose norms are less than $\sigma$.

Proof. By the definitions of $\Phi, \Psi$, and $J$, we know that $\Psi^{\prime}$ is compact, and $\Phi$ is weakly lower semi-continuous and bounded on each bounded subset of $X$. From Lemma 3.1 we can see that $\left(\Phi^{\prime}\right)^{-1}$ is well defined, from condition (A), and $J$ is well defined and continuously Gâteaux differentiable on $X$, with compact derivative. Then we can use Theorem A to obtain the result. Now we show that the hypotheses of Theorem A are fulfilled.

From Lemma 3.2, we can see (i) is satisfied.

From (7) we know that

$$
\sup _{x \in \bar{\Omega}}|u(x)| \leq k\|u\|, \forall u \in X .
$$

Hence, if we let $r=\frac{1}{p^{+}}\left(\frac{c}{k}\right)^{p^{+}}$, for each $u \in X$ such that

$$
\Phi(u) \leq r
$$

by (5), (6) and the assumption $k>c$, we have

$$
\begin{aligned}
\sup _{x \in \bar{\Omega}}|u(x)| & \leq k\|u\| \\
& \leq k \max \left\{\left(\int_{\Omega}|\triangle u(x)|^{p(x)} d x\right)^{\frac{1}{p^{+}}},\left(\int_{\Omega}|\triangle u(x)|^{p(x)} d x\right)^{\frac{1}{p^{-}}}\right\} \\
& \leq k\left(r p^{+}\right)^{\frac{1}{p^{+}}} \\
& \leq c .
\end{aligned}
$$


Due to Lemma 3.3, there exists $u^{*} \in X$ such that

$$
\Phi\left(u^{*}\right)>r>0
$$

and

$$
m(\Omega) \max _{(x, t) \in \bar{\Omega} \times[-c, c]} F(x, t) \leq r p^{-} \frac{\int_{\Omega} F\left(x, u^{*}\right) d x}{\int_{\Omega}|\triangle u(x)|^{p(x)} d x} .
$$

Let $u_{1}(x)=u^{*}(x)$ on $\Omega$, and then by (14) we have

$$
\begin{aligned}
\sup _{u \in \Phi^{-1}((-\infty, r])}(-\Psi(u)) & \leq \int_{\Omega\{u \mid \Phi(u) \leq r\}} \sup F(x, u) d x \\
& \leq \int_{\Omega} \sup _{|t| \leq c} F(x, t) d x \\
& <m(\Omega) \max _{(x, t) \in \bar{\Omega} \times[-c, c]} F(x, t) d x \\
& \leq r p^{-} \frac{\int_{\Omega} F\left(x, u_{1}\right) d x}{\int_{\Omega}\left|\triangle u_{1}(x)\right|^{p(x)} d x} \\
& \leq r \frac{-\Psi\left(u_{1}\right)}{\Phi\left(u_{1}\right)}
\end{aligned}
$$

Fixing any $h>1$, it is easy to see that

$$
\sup _{u \in \Phi^{-1}((-\infty, r])}(-\Psi(u))+\frac{r \frac{-\Psi\left(u_{1}\right)}{\Phi\left(u_{1}\right)}-\sup _{u \in \Phi^{-1}((-\infty, r])}(-\Psi(u))}{h}<r \frac{-\Psi\left(u_{1}\right)}{\Phi\left(u_{1}\right)} .
$$

By Proposition 1.3 of [3], when $\rho$ satisfies

$$
\sup _{u \in \Phi^{-1}((-\infty, r])}(-\Psi(u))+\frac{r \frac{-\Psi\left(u_{1}\right)}{\Phi\left(u_{1}\right)}-\sup _{u \in \Phi^{-1}((-\infty, r])}(-\Psi(u))}{h}<\rho<r \frac{-\Psi\left(u_{1}\right)}{\Phi\left(u_{1}\right)}
$$

we have

$$
\sup _{\lambda \in R} \inf _{u \in X}(\Phi(u)+\lambda(\rho+\Psi(u)))<\inf _{u \in X} \sup _{\lambda \in\left[0, \alpha_{1}\right]}(\Phi(u)+\lambda(\rho+\Psi(u))),
$$

where $\alpha_{1}=\frac{h r}{r \frac{-\Psi\left(u_{1}\right)}{\Phi\left(u_{1}\right)}-\sup _{u \in \Phi^{-1}((-\infty, r])}(-\Psi(u))}>0$.

Then (ii) of Theorem A holds with $I=\left[0, \alpha_{1}\right]$. Then all the hypotheses of Theorem A are fulfilled. By Theorem A, we know that there exist an open interval $\Lambda \subseteq I$ and a positive constant $\sigma$ such that for any $\lambda \in \Lambda$, there exists $\delta>0$ and for each $\mu \in[0, \delta]$, problem (1) has at least three weak solutions whose norms are less than $\sigma$.

Acknowledgements. The authors thank the referees for valuable comments which have led to an improvement of the presentation of this paper. 


\section{References}

1] A. El Amrouss, F. Moradi, and M. Moussaoui, Existence of solutions for fourth-order PDEs with variable exponents, Electron. J. Differential Equations 2009 (2009), no. 153, $1-13$.

[2] A. Ayoujil and A. R. El Amrouss, On the spectrum of a fourth order elliptic equation with variable exponent, Nonlinear Anal. 71 (2009), no. 10, 4916-4926.

[3] G. Bonanno, Some remarks on a three critical points theorem, Nonlinear Anal. 54 (2003), no. 4, 651-665

[4] X. L. Fan, Solutions for $p(x)$-Laplacian Dirichlet problems with singular coefficients, J. Math. Anal. Appl. 312 (2005), no. 2, 464-477.

[5] X. L. Fan and D. Zhao, On the spaces $L^{p(x)}(\Omega)$ and $W^{m, p(x)}(\Omega)$, J. Math. Anal. Appl. 263 (2001), no. 2, 424-446.

[6] H. Hudzik, On generalized Orlicz-Sobolev space, Funct. Approx. Comment. Math. 4 (1976), 37-51.

[7] O. Kováčik and J. Rákosník, On spaces $L^{p(x)}(\Omega)$ and $W^{k, p(x)}(\Omega)$, Czechoslovak Math. J. 41 (1991), no. 4, 592-618.

[8] S. Kichenassamy and L. Véron, Singular solutions of the p-Laplace equation, Math. Ann. 275 (1986), no. 4, 599-615.

[9] C. Li and C. L. Tang, Three solutions for a Navier boundary value problem involving the p-biharmonic, Nonlinear Anal. 72 (2010), no. 3-4, 1339-1347.

[10] S. Liu and M. Squassina, On the existence of solutions to a fourth-order quasilinear resonant problem, Abstr. Appl. Anal. 7 (2002), no. 3, 125-133.

[11] B. Ricceri, A three critical points theorem revisited, Nonlinear Anal. 70 (2009), no. 9, 3084-3089.

[12] M. Rúzicka, Electro-rheological Fluids: Modeling and Mathematical Theory, Lecture Notes in Math., vol. 1784, Springer-Verlag, Berlin, 2000.

[13] S. G. Sanko, Denseness of $C_{0}^{\infty}\left(R^{N}\right)$ in the generalized Sobolev spaces $W^{m, p(x)}\left(R^{N}\right)$, Dokl. Akad. Nauk 369 (1999), no. 4, 451-454.

[14] A. Zang and Y. Fu, Interpolation inequalities for derivatives in variable exponent Lebesgue-Sobolev spaces, Nonlinear Anal. 69 (2008), no. 10, 3629-3636.

[15] E. Zeidler, Nonlinear Functional Analysis and its Applications. II/B, Nonlinear Monotone Operators, Springer, New York, 1990.

HONGHUI YIN

School of Mathematical Sciences

HuAIYIN NORMAL UNIVERSITY

Jiangsu Huaian 223001, P. R. China

E-mail address: yinhh@hytc.edu.cn

YING LIU

School of Mathematical Sciences

HuAiYin Normal UNIVERSITY

Jiangsu Huaian 223001, P. R. China

E-mail address: liuying1032032115@126.com 